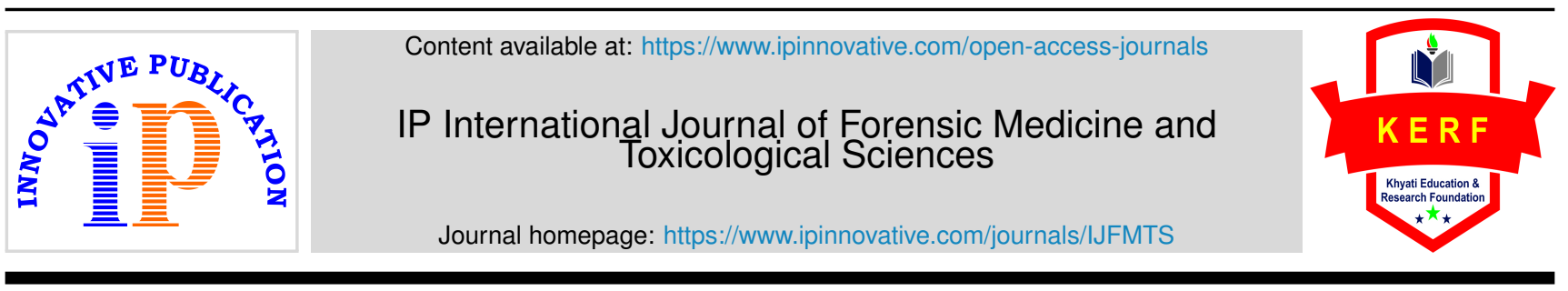

Original Research Article

\title{
Analysis of vitreous humour in determining postmortem interval (time since death) - A prospective study
}

\author{
S. Angayarkanni ${ }^{1} *$ \\ ${ }^{1}$ Institute of Forensic Medicine, Madras Medical College, Chennai, Tamil Nadu, India
}

\section{A R T I C L E I N F O}

\section{Article history:}

Received 04-11-2020

Accepted 05-12-2020

Available online 07-01-2021

\section{Keywords:}

Autopsy

potassium

time since death

vitreous humour

\begin{abstract}
A B S T R A C T
Aim: Aim of the study was to find out post-mortem interval (time since death) on 100 cases which were brought for medico-legal autopsy at Institute of Forensic Medicine and Toxicology, Rajiv Gandhi Government General Hospital, Madras Medical College, Chennai by analyzing changes in biochemical markers in vitreous humour of both eyes.

Materials and Methods : Two samples of vitreous humour were taken from both eyes, first sample immediately after the body was received in the mortuary and second sample at the time of starting postmortem examination. The samples were sent to the Department of Biochemistry for analyzing sodium and potassium concentration.

Observations: After the statistical analysis it was observed that $\mathrm{p}<0.001$ which is a statistically significant data and concludes positive correlation of time since death with potassium increase whereas sodium falls as time increases which is not statistically significant. The study also showed the regression for vitreous samples with time since death as a dependent variable and depicts a linear correlation between time since death and increase in potassium. The $\mathrm{T}$ test compared values of sodium and potassium and it showed $\mathrm{p}>0.05$ which concludes there is no significant difference.

Conclusion: It was concluded that there was a linear correlation between time since death and potassium levels in vitreous samples from both sides at different time intervals. There is no significant difference in values of samples from both sides. The study also concludes that sodium values are not statistically significant and shows negative correlation with time since death.
\end{abstract}

(C) This is an open access article distributed under the terms of the Creative Commons Attribution License (https://creativecommons.org/licenses/by/4.0/) which permits unrestricted use, distribution, and reproduction in any medium, provided the original author and source are credited.

\section{Introduction}

'Forensic Medicine' or 'Legal Medicine' is the application of knowledge and principles of medicine for the purpose of law, both civil and criminal. Its main objective is to aid in the administration of justice. ${ }^{1}$

Forensic experts summoned to the scene of death are aimed to provide an estimation of the time elapsed since death. In forensic medicine and thereby criminal law, the important problem is to estimate the time of death. In the court of law, a medical practitioner has to give evidence as a medical jurist in order to prove the innocence or guilt of an accused. He has a great responsibility that he may be the

\footnotetext{
* Corresponding author.

E-mail address: dr.angai5774kayal@gmail.com (S. Angayarkanni).
}

only reliable evidence in the court of law. ${ }^{2}$

Post mortem interval is the interval between the death and time of examination of a body. This is important in knowing when the crime was committed. It helps police to start their inquiries with the available information and also in dealing the cases more efficiently. It also helps in including and excluding the suspects and culprits and in confirming the statements of the suspect. Estimation of time since death is useful in civil cases such as inheritance of property, insurance claims etc. ${ }^{3}$

Longer the post mortem interval, wider will be the limits of probability. Though the changes like cooling of body, eye changes, post-mortem staining, rigor mortis, stomach contents, bladder and bowel contents, decomposition changes and circumstantial evidence can sometimes yield 
a reasonable accurate result in early post-mortem hours, they are not reliable because of environmental factors. Hence forensic pathologists and biochemists have been concentrating on biochemical changes that occur in body fluids such as blood and compartmental fluids like vitreous humour, cerebrospinal fluid, pleural fluid, pericardial fluid and synovial fluid. ${ }^{4}$

Because of the lack of oxygen in the circulation, alteration in the enzymatic reaction and stoppage of production of metabolites, extensive biochemical changes occur in all body fluids. Such changes provide chemical markers which help to determine time of death accurately. ${ }^{5}$

These metabolites are products of metabolism and intermediates of smaller molecular size which have functions in normal growth and development of cells. They may be exogenous or endogenous changes and hence metabolite profiles are altered following death. ${ }^{6}$

The changes that occur after death have been identified and attributed to agonal period, changes in the early postmortem period and diffusion of substances between various body fluids.

Biochemical markers are divided into two classes metabolites and proteins. Sodium, potassium, chloride, calcium, magnesium, phosphate, lactic acid, hypoxanthine, urea, creatinine, uric acid, ammonia, catecholamines, ethanol are metabolites. Other class includes total proteins and enzymes aspartate aminotransferase and lactate dehydrogenase.

Immediately after death, the changes that occur in the chemical composition of body fluids such as blood, vitreous humour, synovial fluid and cerebrospinal fluid are described by thanatochemistry. After death, the electrolytes and the chemicals redistribute, cellular integrity is lost, energy dependent trans membrane transportation is absent. Hence it will be difficult to assess post-mortem blood samples. Because of the breakdown of the active membrane transport and rapid breakdown of metabolism after death, only stable analytes can be estimated in blood samples. ${ }^{7}$

Among the various body fluids like blood, serum, cerebrospinal fluid, aqueous humour, synovial fluid and vitreous humour, estimation of concentration of potassium in vitreous humour is the most widely used method. ${ }^{8}$

Vitreous fluid is an acellular , transparent, inert, colourless, hydrophilic viscous fluid that is present between the lens and retina within the eyeball which is an important supporting structure that serves the optical function. Its weight is approximately 4 grams and its volume is approximately $4 \mathrm{cc}$. It is composed of $99 \%$ of water with soluble proteins, amino acids, low molecular weight constituents, glucose, type II collagen, hyaluronic acid, inorganic salts and ascorbic acid. ${ }^{9,10}$

It is more than 40 years that the biochemical changes in vitreous have been on analysis. The underlying principle in analysing vitreous humour is that it is a closed compartment which is separated from the rest of the body. But ambient temperature may influence.

The composition of the vitreous is closely related to that of serum, aqueous humour and cerebrospinal fluid. It is relatively stable, easily accessible, less susceptible to rapid chemical changes, well protected from decomposition and contamination. Hence it is more suitable for analysis than other fluids in estimating time since death. ${ }^{11}$

Eye and thereby Vitreous humour is well protected even in case of severe head injury and in burns. This was frequently remarked as "miraculous Escape". ${ }^{12}$

Many studies have been conducted on analysis of sodium and potassium in vitreous humour. ${ }^{13-15}$

Concentration of sodium, potassium and urate were analysed in vitreous humour of both the eyes at the same time of death showed variation between the eyes. ${ }^{16}$

Many studies have shown that the vitreous potassium level increases with increase in post-mortem interval. ${ }^{4,16-41}$

There was a linear relationship of increase in potassium concentration with increase in postmortem interval. ${ }^{42}$

As the post mortem interval increases, the potassium also increases whereas there is negative correlation with sodium and no correlation with chloride and calcium. ${ }^{43}$

Sodium concentration in normal body is 136-145 $\mathrm{mEq} / \mathrm{L}$.Potassium concentration in normal body is $50-55$ $\mathrm{mEq} / \mathrm{kg}$ of body weight of which $160 \mathrm{mmol} / \mathrm{l}$ ie. $98 \%$ of the potassium exists within cell whereas extracellular concentration is $3.5-5.5 \mathrm{mmol} / 1 .{ }^{44}$ The normal sodium value in vitreous humour is $118-124 \mathrm{mmol} / \mathrm{L}$ and potassium value in vitreous humour is $2.6-4.2 \mathrm{mmol} / \mathrm{L} .{ }^{4}$ Potassium is actively transported from ciliary body into anterior vitreous and posterior chamber. Potassium is also contributed by the lens. ${ }^{44}$

\section{Aims and Objectives}

1. To study the use of vitreous humour in finding postmortem interval

2. To compare the distribution of substances in between two eyes

3. To determine post-mortem interval even in decomposed and charred bodies

\section{Materials and Methods}

This study was carried out on 100 cases which were brought for medico-legal autopsy at Rajiv Gandhi Government General Hospital, Madras Medical College, Chennai-3. Cases which were admitted in hospital and whose exact times of death were known were selected for the study. Details of these cases were obtained from the hospital records, police records, relatives and friends. Cases whose exact time of death was not known and with previous history of eye or orbital injury or surgery, posterior segment diseases were excluded from the study. 
Vitreous humour was collected from both eyes. First sample of vitreous humour was aspirated from both the eyes simultaneously as early as possible after the entry of the body into mortuary. Second sample was taken from both the eyes simultaneously at the time of post-mortem examination. The samples which were turbid and mixed with blood were discarded. The details of the cases were recorded.

Samples of vitreous humour were collected from the posterior chamber by aspirating gradually and slowly through a puncture 5-6 $\mathrm{mm}$ away from the limbus using a sterile 20 gauge needle taking care to avoid tearing of any loose tissue fragments surrounding the vitreous chamber.

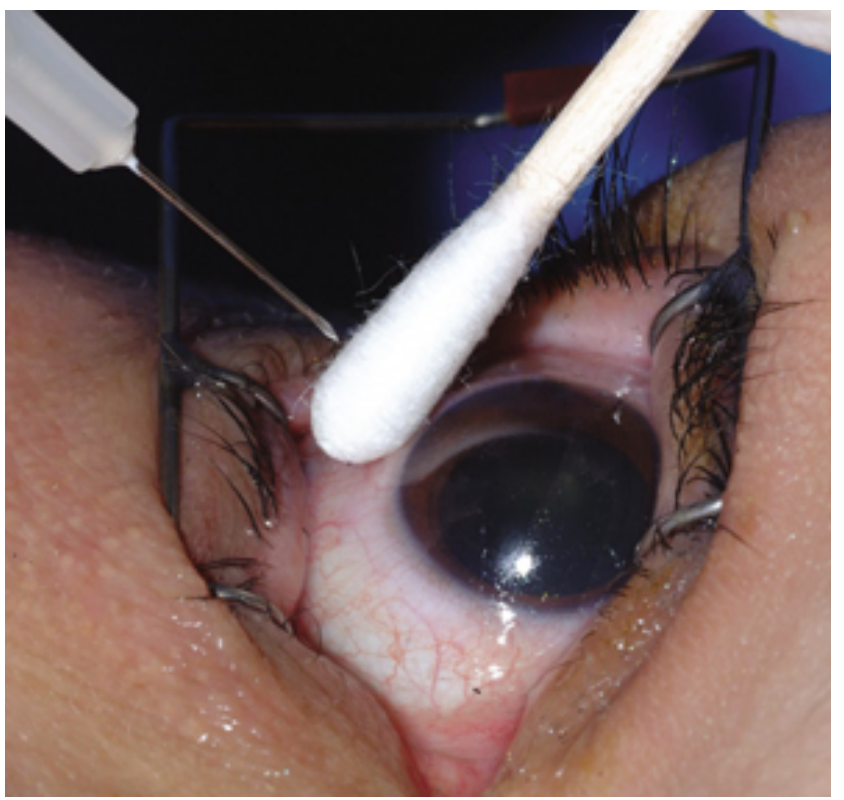

Fig. 1: Procedure of Vitreous aspiration

The samples were sent to the Institute of Biochemistry, Rajiv Gandhi Government General Hospital, Madras Medical College, Chennai-3.

The samples were analysed by Medica Easylyte Sodium/potassium analyser. It is a fully automated, electrolyte system that is microprocessor controlled. It uses Ion Selective Electrode(ISE) technology for the measurement of electrolytes in which are stored, an easily accessible quality control data. It measures sodium, potassium, chloride, lithium, calcium and $\mathrm{pH}$ of the serum, whole blood, plasma, urine, vitreous and synovial fluid. The calibrants are packed in a convenient solution pack with

Standard A solution, $800 \mathrm{ml}(140.0 \mathrm{mmol} / \mathrm{L}$ sodium $* 4.0$ $\mathrm{mmol} / \mathrm{L}$ potassium *Buffer* Preservative * wetting agent).,

Standard B solution $180 \mathrm{ml}(35.0 \mathrm{mmol} / \mathrm{L}$ sodium, 16.0 $\mathrm{mmol} / \mathrm{L}$ potassium *Buffer* Preservative*Wetting agent)

Wash solution, $80 \mathrm{ml}(0.1 \mathrm{~mol} / \mathrm{L}$ (Ammonium bi fluoride)
Ion selective Electrode method consists of a thin membrane across which only the intended ion can be transported. The transport of ions from a high concentration to a low concentration through a selective binding with some sites within the membrane creates a potential difference which is measured in Volts.

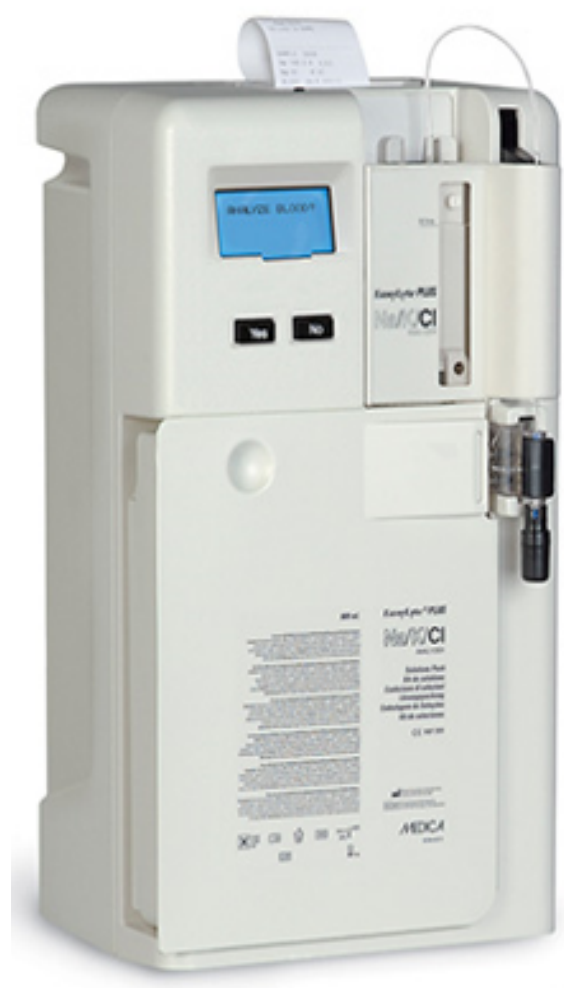

Fig. 2: Medica Easylyte Electrolyte Analyzer

\section{Observations}

This study was carried out on 100 cases whose time of death was known who were brought to the mortuary, Rajiv Gandhi Government General Hospital, Madras Medical College, Chennai-3 for post-mortem examination.

Samples of vitreous humour were taken at intervals. First sample was taken as early as possible after the entry of the dead bodies into the mortuary from both the eyes. Second sample was taken at the time of post-mortem examination from both the eyes.

Only the clear vitreous humour was analysed. Turbid or blood stained samples were excluded from the study.

The samples were analysed for sodium and potassium by selective ion electrode method.

This Table 1 shows percentage of cases depending upon time since death in sample 1 and sample 2 of vitreous humour of both eyes.

Table 1 shows number and percentage of cases from sample 1 and 2 which falls within the time intervals. It was 
Table 1: Percentage of cases depending upon Time since death

\begin{tabular}{lccccc}
\hline S.No & Time since Death & \multicolumn{2}{c}{ Sample I } & \multicolumn{2}{c}{ Sample II } \\
(hours ) & Number of cases & $\begin{array}{c}\text { Percentage of } \\
\text { cases }\end{array}$ & Number of cases & \\
Percentage of cases \\
1. & Within 12 hours & 53 & $53 \%$ & 31 & $31 \%$ \\
2. & $12.1-24$ hours & 40 & $40 \%$ & 57 & $57 \%$ \\
3. & Above 24 hours & 7 & $7 \%$ & 12 & $12 \%$ \\
& Total & 100 & $100 \%$ & 100 & $100 \%$ \\
\hline
\end{tabular}

Table 2: Descriptive for Sample I \& II - Time since death

\begin{tabular}{|c|c|c|c|c|c|c|c|c|c|c|}
\hline \multirow{5}{*}{$\begin{array}{l} \\
\text { Sample } \\
\text { I } \\
\text { R Eye } \\
\mathrm{Na}\end{array}$} & \multirow[t]{2}{*}{$\begin{array}{l}P \\
\text { value }\end{array}$} & \multirow{2}{*}{$\begin{array}{l}\text { Time } \\
\text { since } \\
\text { death }\end{array}$} & \multirow[t]{2}{*}{$\mathbf{N}$} & \multirow[t]{2}{*}{ Mean } & \multirow[t]{2}{*}{$\begin{array}{l}\text { Std. } \\
\text { Deviation }\end{array}$} & \multirow[t]{2}{*}{$\begin{array}{l}\text { Std. } \\
\text { Error }\end{array}$} & \multicolumn{2}{|c|}{$\begin{array}{l}\text { 95\% Confidence Interval for } \\
\text { Mean }\end{array}$} & \multirow[t]{2}{*}{ Min } & \multirow[t]{2}{*}{$\operatorname{Max}$} \\
\hline & & & & & & & Lower Bound & Upper Bound & & \\
\hline & \multirow{4}{*}{$\mathrm{P}<0.001$} & $<12$ hrs & 53 & \multirow{2}{*}{\multicolumn{2}{|c|}{$\begin{array}{l}139.66239 .51061 \\
145.27257 .76732\end{array}$}} & 1.30638 & 137.0408 & 142.2837 & 126.50 & 157.90 \\
\hline & & $\begin{array}{l}12-24 \\
\text { hrs }\end{array}$ & 40 & & & 1.22812 & 142.7884 & 147.7566 & 125.10 & 158.70 \\
\hline & & $>24 \mathrm{hrs}$ & 7 & \multirow{2}{*}{\multicolumn{2}{|c|}{$\begin{array}{l}137.35717 .32481 \\
141.74509 .12358\end{array}$}} & 2.76852 & 130.5828 & 144.1315 & 125.70 & 147.90 \\
\hline & & Total & 100 & & & .91236 & 139.9347 & 143.5553 & 125.10 & 158.70 \\
\hline \multirow{4}{*}{$\begin{array}{l}\text { Sample } \\
\text { I } \\
\text { R Eye } \\
\text { K }\end{array}$} & \multirow{4}{*}{$\mathrm{P}<0.001$} & $<12$ hrs & 53 & 6.9509 & 2.85628 & .39234 & 6.1637 & 7.7382 & 2.90 & 18.60 \\
\hline & & $\begin{array}{l}12-24 \\
\mathrm{hrs}\end{array}$ & 40 & 8.9075 & 1.76394 & .27890 & 8.3434 & 9.4716 & 5.80 & 14.60 \\
\hline & & $>24 \mathrm{hrs}$ & 7 & 11.1143 & 3.74941 & 1.41714 & 7.6467 & 14.5819 & 7.20 & 16.30 \\
\hline & & Total & 100 & 8.0250 & 2.82311 & .28231 & 7.4648 & 8.5852 & 2.90 & 18.60 \\
\hline \multirow{4}{*}{$\begin{array}{l}\text { Sample } \\
\text { I } \\
\text { Lt Eye } \\
\mathrm{Na}\end{array}$} & \multirow{4}{*}{$\mathrm{P}<0.001$} & $<12 \mathrm{hrs}$ & 31 & \multirow{2}{*}{\multicolumn{2}{|c|}{$\begin{array}{l}142.09359 .18633 \\
141.83169 .26536\end{array}$}} & 1.64991 & 138.7240 & 145.4631 & 126.30 & 158.00 \\
\hline & & $\begin{array}{l}12-24 \\
\mathrm{hrs}\end{array}$ & 57 & & & 1.22723 & 139.3731 & 144.2900 & 124.80 & 159.00 \\
\hline & & $>24 \mathrm{hrs}$ & 12 & \multirow{2}{*}{\multicolumn{2}{|c|}{$\begin{array}{l}141.27508 .20179 \\
141.84609 .03689\end{array}$}} & 2.36765 & 136.0638 & 146.4862 & 126.00 & 151.80 \\
\hline & & Total & 100 & & & .90369 & 140.0529 & 143.6391 & 124.80 & 159.00 \\
\hline \multirow{4}{*}{$\begin{array}{l}\text { Sample } \\
\text { I Lt } \\
\text { Eye } \\
\text { K }\end{array}$} & \multirow{4}{*}{$\mathrm{P}<0.001$} & $<12$ hrs & 31 & 7.9323 & 3.21894 & .57814 & 6.7515 & 9.1130 & 3.10 & 18.70 \\
\hline & & $\begin{array}{l}12-24 \\
\mathrm{hrs}\end{array}$ & 57 & 7.4649 & 2.30732 & .30561 & 6.8527 & 8.0771 & 3.20 & 14.90 \\
\hline & & $>24 \mathrm{hrs}$ & 12 & 9.9333 & 2.79686 & .80738 & 8.1563 & 11.7104 & 6.90 & 14.90 \\
\hline & & Total & 100 & 7.9060 & 2.76240 & .27624 & 7.3579 & 8.4541 & 3.10 & 18.70 \\
\hline \multirow{4}{*}{$\begin{array}{l}\text { Sample } \\
2 \\
\mathrm{R} \text { Eye } \\
\mathrm{Na}\end{array}$} & \multirow{4}{*}{$\mathrm{P}<0.001$} & $<12 \mathrm{hrs}$ & 31 & \multirow{2}{*}{\multicolumn{2}{|c|}{$\begin{array}{l}140.69687 .21722 \\
146.32987 .04151\end{array}$}} & 1.29625 & 138.0495 & 143.3441 & 130.60 & 156.70 \\
\hline & & $\begin{array}{l}12-24 \\
\mathrm{hrs}\end{array}$ & 57 & & & .93267 & 144.4615 & 148.1982 & 128.50 & 165.80 \\
\hline & & $>24 \mathrm{hrs}$ & 12 & & 2.16688 & 134.8724 & 144.4109 & 126.80 & 149.40 \\
\hline & & Total & 100 & \multicolumn{2}{|c|}{143.78107 .67383} & .76738 & 142.2583 & 145.3037 & 126.80 & 165.80 \\
\hline \multirow{4}{*}{$\begin{array}{l}\text { Sample } \\
2 \mathrm{R} \\
\text { Eye } \\
\mathrm{K}\end{array}$} & & $<12$ hrs & 31 & 7.7839 & 1.88186 & .33799 & 7.0936 & 8.4741 & 4.90 & 14.70 \\
\hline & $\mathrm{P}<0.001$ & $\begin{array}{l}12-24 \\
\mathrm{hrs}\end{array}$ & 57 & 9.9561 & 2.17420 & .28798 & 9.3792 & 10.5330 & 6.80 & 19.10 \\
\hline & & $>24 \mathrm{hrs}$ & 12 & 12.8917 & 3.49089 & 1.00773 & 10.6737 & 15.1097 & 7.90 & 18.10 \\
\hline & & Total & 100 & 9.6350 & 2.74209 & .27421 & 9.0909 & 10.1791 & 4.90 & 19.10 \\
\hline Sample & & $<12 \mathrm{hrs}$ & 31 & 140.767 & 77.30823 & 1.31260 & 138.0871 & 143.4484 & 131.00 & 156.40 \\
\hline L Eye & $\mathrm{P}<0.001$ & $\begin{array}{l}12-24 \\
\text { hrs }\end{array}$ & 57 & 146.487 & 76.89241 & .91292 & 144.6589 & 148.3165 & 128.30 & 165.30 \\
\hline $\mathrm{Na}$ & & $>24 \mathrm{hrs}$ & 12 & 140.225 & 06.81604 & 1.96762 & 135.8943 & 144.5557 & 126.30 & 149.20 \\
\hline & & Total & 100 & 143.9630 & 07.53521 & .75352 & 142.4679 & 145.4581 & 126.30 & 165.30 \\
\hline & & $<12 \mathrm{hrs}$ & 31 & 7.6129 & 1.77759 & .31926 & 6.9609 & 8.2649 & 5.20 & 13.90 \\
\hline $\begin{array}{l}\text { Sample2 } \\
\text { L Eye }\end{array}$ & $\mathrm{P}<0.001$ & $\begin{array}{l}12-24 \\
\mathrm{hrs}\end{array}$ & 57 & 9.8018 & 2.13897 & .28331 & 9.2342 & 10.3693 & 6.80 & 18.90 \\
\hline & & $>24 \mathrm{hrs}$ & 12 & 12.5250 & 3.29162 & .95021 & 10.4336 & 14.6164 & 7.50 & 17.70 \\
\hline & & Total & 100 & 9.4500 & 2.65020 & .26502 & 8.9241 & 9.9759 & 5.20 & 18.90 \\
\hline
\end{tabular}


Table 3: Regression co-efficients for Vitreous Fluid

\section{Coefficients $^{a}$}

Model

$\begin{array}{llll} & & \text { B } & \text { Std. Error } \\ \text { Sample 1 } & \text { (Constant) } & .740 & .170 \\ & \text { Right K Vitreous } & .100 & .020 \\ & \text { humour } & .781 & .175 \\ \text { (Constant) } & .096 & .021 \\ & \text { Left K Vitreous } & & \\ \text { humour } & .548 & .189 \\ \text { (Constant) } & .131 & .019 \\ \text { Sample 2 } & \text { Right K Vitreous } & .535 & .194 \\ & \text { humour } & .135 & .020 \\ & \text { (Constant) } & & \end{array}$

$\begin{aligned} & \text { Standardized } \\ & \text { Coefficients } \\ & \text { Beta }\end{aligned}$
.450
.421
.574
.567

$\mathbf{t}$

Sig.

$\begin{array}{ll}4.355 & .000 \\ 4.986 & .000 \\ & \\ 4.463 & .000 \\ 4.592 & .000 \\ & \\ 2.899 & .005 \\ 6.940 & .000 \\ & .007 \\ 2.751 & .000 \\ 6.816 & \end{array}$

a. Dependent Variable: TIME SINCE DEATH

Table 4: Correlation between TSD and $\mathrm{K}$ and $\mathrm{Na}$ Values

\begin{tabular}{|c|c|c|c|c|c|c|c|c|c|}
\hline \multirow{5}{*}{ TSD } & & $\begin{array}{l}\text { Right Na } \\
1\end{array}$ & Right K 1 & Left Na 1 & Left $\mathrm{K} 1$ & $\begin{array}{l}\text { Right Na } \\
2\end{array}$ & Right K 2 & Left $\mathrm{Na} 2$ & Left $\mathrm{K} 2$ \\
\hline & Pearson & .004 & $.580^{* *}$ & .004 & $.536^{H H}$ & -.195 & $.611^{* *}$ & -.173 & $.581^{* *}$ \\
\hline & Correlation & & & & & & & & \\
\hline & P Value & .967 & .000 & .969 & .000 & .052 & .000 & .088 & .000 \\
\hline & $\mathrm{N}$ & 100 & 100 & 100 & 100 & 100 & 100 & 99 & 100 \\
\hline
\end{tabular}

**. Correlation is significant at the 0.01 level (2-tailed).

*. Correlation is significant at the 0.05 level (2-tailed).

Table 5: Group statistics

\begin{tabular}{llllll}
\hline & Group & N & Mean & Std. Deviation & Std. Error Mean \\
\multirow{2}{*}{ sample I Eye Vitreous Sodium } & Right & 100 & 141.7450 & 9.12358 & .91236 \\
\multirow{2}{*}{ sample I Eye Vitreous } & Left & 100 & 141.8460 & 9.03689 & .90369 \\
Potassium & Right & 100 & 8.0250 & 2.82311 & .28231 \\
\multirow{2}{*}{ sample II Eye Vitreous Sodium } & Left & 100 & 7.9060 & 2.76240 & .27624 \\
sample II Eye Vitreous & Right & 100 & 143.7810 & 7.67383 & .76738 \\
Potassium & Left & 100 & 143.9630 & 7.53521 & .75352 \\
& Right & 100 & 9.6350 & 2.74209 & .27421 \\
\hline
\end{tabular}

$\mathrm{P}>0.05$ no difference among left and right values

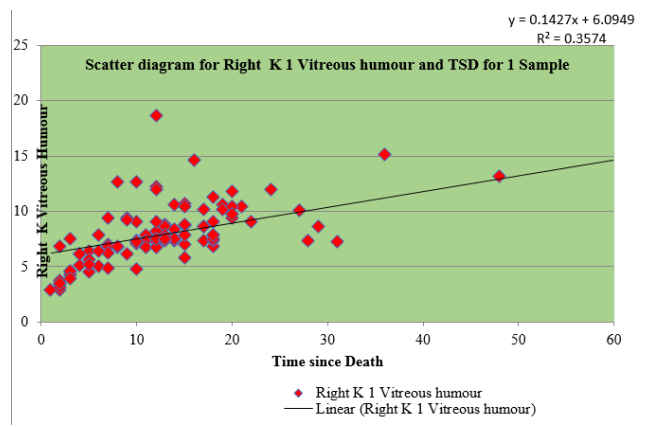

Fig. 3: Correlation of sample I of right vitreous potassium with time since death

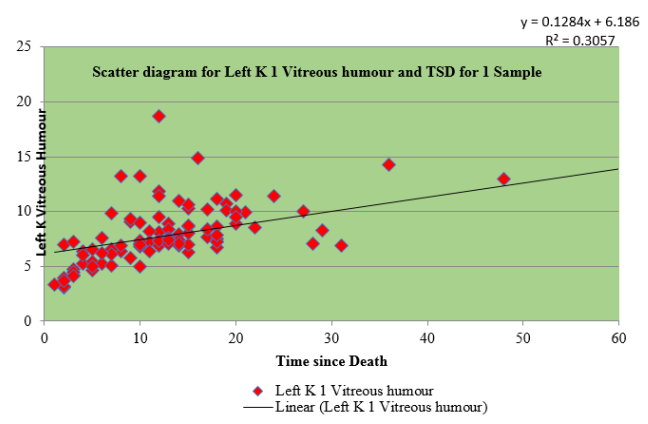

Fig. 4: Correlation of sample I of left vitreous potassium with time since death 
Table 6: T-Tests

\begin{tabular}{|c|c|c|c|c|c|c|c|}
\hline \multirow{10}{*}{$\begin{array}{l}\text { T - Test } \\
\text { for timing } \\
0-12 \\
\text { hours }\end{array}$} & \multicolumn{7}{|l|}{ Group Statistics } \\
\hline & & P value & Group & $\mathbf{N}$ & Mean & Std. Deviation & Std. Error Mean \\
\hline & \multirow{2}{*}{$\begin{array}{l}\text { Left Na Vitreous } \\
\text { humour }\end{array}$} & \multirow{2}{*}{$\mathrm{p}>0.05$} & Sample 1 & 53 & 139.7415 & 9.38445 & 1.28905 \\
\hline & & & Sample 2 & 31 & 140.7677 & 7.30823 & 1.31260 \\
\hline & \multirow{2}{*}{$\begin{array}{l}\text { Left K Vitreous } \\
\text { humour }\end{array}$} & \multirow{2}{*}{$\mathrm{p}>0.05$} & Sample 1 & 53 & 6.9245 & 2.85986 & .39283 \\
\hline & & & Sample 2 & 31 & 7.6129 & 1.77759 & .31926 \\
\hline & \multirow{2}{*}{$\begin{array}{l}\text { Right } \mathrm{Na} \text { Vitreous } \\
\text { humour }\end{array}$} & \multirow[b]{2}{*}{$\mathrm{p}>0.05$} & Sample 1 & 53 & 139.6623 & 9.51061 & 1.30638 \\
\hline & & & Sample 2 & 31 & 140.6097 & 7.09983 & 1.27517 \\
\hline & \multirow{2}{*}{$\begin{array}{l}\text { Right K Vitreous } \\
\text { humour }\end{array}$} & \multirow[b]{2}{*}{$\mathrm{p}>0.05$} & Sample 1 & 53 & 6.9415 & 2.85727 & .39248 \\
\hline & & & Sample 2 & 31 & 7.6903 & 1.85461 & .33310 \\
\hline \multirow{8}{*}{$\begin{array}{l}\mathrm{T}-\text { Test for } \\
\text { timing } 12- \\
24 \text { hours }\end{array}$} & Left $\mathrm{Na}$ & \multirow{2}{*}{$\mathrm{p}>0.05$} & Sample 1 & 40 & 145.3875 & 7.70588 & 1.21841 \\
\hline & Vitreous humour & & Sample 2 & 57 & 146.4877 & 6.89241 & .91292 \\
\hline & Left $\mathrm{K}$ & \multirow{2}{*}{$\mathrm{P}<0.05^{*}$} & Sample 1 & 40 & 8.7575 & 1.75921 & .27816 \\
\hline & Vitreous humour & & Sample 2 & 57 & 9.8018 & 2.13897 & .28331 \\
\hline & Right $\mathrm{Na}$ & \multirow{2}{*}{$\mathrm{p}>0.05$} & Sample 1 & 40 & 145.2725 & 7.76732 & 1.22812 \\
\hline & Vitreous humour & & Sample 2 & 57 & 146.3298 & 7.04151 & .93267 \\
\hline & Right K & \multirow{2}{*}{$\mathrm{P}<0.05^{*}$} & Sample 1 & 40 & 8.9075 & 1.76394 & .27890 \\
\hline & Vitreous humour & & Sample 2 & 57 & 9.9561 & 2.17420 & .28798 \\
\hline \multirow{8}{*}{$\begin{array}{l}\mathrm{T}-\text { Test for } \\
\text { timing } \\
\text { more than } \\
24 \text { hours }\end{array}$} & Left $\mathrm{Na}$ & \multirow{2}{*}{$\mathrm{p}>0.05$} & Sample 1 & 7 & 137.5429 & 7.34231 & 2.77513 \\
\hline & Vitreous humour & & Sample 2 & 12 & 140.2250 & 6.81604 & 1.96762 \\
\hline & Left $\mathrm{K}$ & \multirow{2}{*}{$\mathrm{p}>0.05$} & Sample 1 & 7 & 10.6429 & 3.40385 & 1.28653 \\
\hline & Vitreous humour & & Sample 2 & 12 & 12.5250 & 3.29162 & .95021 \\
\hline & Right $\mathrm{Na}$ & \multirow{2}{*}{$\mathrm{p}>0.05$} & Sample 1 & 7 & 137.3571 & 7.32481 & 2.76852 \\
\hline & Vitreous humour & & Sample 2 & 12 & 139.6417 & 7.50630 & 2.16688 \\
\hline & Right K & \multirow{2}{*}{$\mathrm{p}>0.05$} & Sample 1 & 7 & 11.1143 & 3.74941 & 1.41714 \\
\hline & Vitreous humour & & Sample 2 & 12 & 12.8917 & 3.49089 & 1.00773 \\
\hline
\end{tabular}

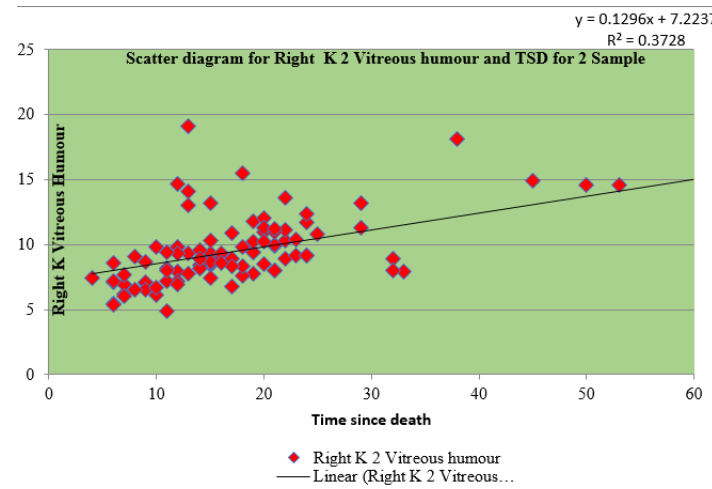

Fig. 5: Correlation of sample II of right vitreous potassium with time since death

concluded that in sample 1 , there were 53 cases with time since death within 12 hours which constituted $53 \%$, there were 40 cases with time since death from 12.1 to 24 hours which constituted $40 \%$ and 7 cases with time since death more than 24 hours which constituted $7 \%$ out of 100 total cases. In sample 2 , there were 31 cases with time since death within 12 hours which constituted $31 \%$, there were 57 cases with time since death from 12.1 to 24 hours which constituted 57\% and 12 cases with time since death more than 24 hours which constituted $12 \%$ out of 100 total cases.

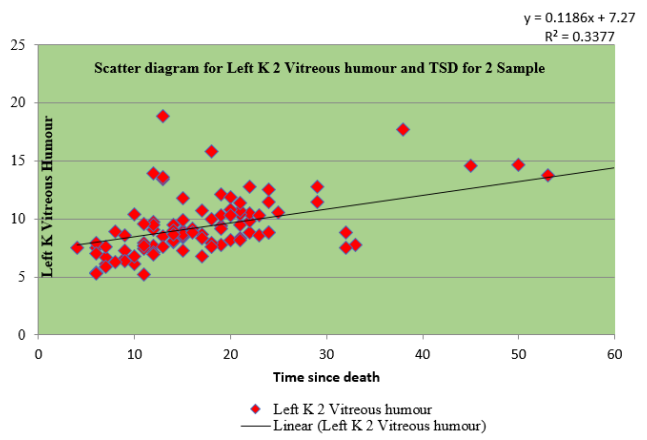

Fig. 6: Correlation of sample II of left vitreous potassium with time since death

Table 2 shows total number of cases N, Mean, Standard deviation, standard error, $95 \%$ confidence interval for mean and the range of sodium and potassium values of sample I \& II of right and left eye. Each group have different values ( $<12$ hours, 12.1-24 hours and $>24$ hours). The table depicts $\mathrm{p}<0.001$ which is a statistically significant data which concludes the positive correlation of time since death with potassium increase whereas sodium level falls as time increases. Hence it is not statistically significant.

Table 3 shows the regression for sample I and II of right and left eye vitreous fluid with time since death as a dependent variable. The values of vitreous potassium 
Table 7: Oneway ANOVA for Comparing Left and Right Na and K values for Three periods ( 0 to12, 12 to 24 and Above 24 hrs Sample $1 \&$ II

\begin{tabular}{|c|c|c|c|c|c|c|c|c|c|c|}
\hline Sample & $\begin{array}{l}\text { Time since } \\
\text { death }\end{array}$ & $\mathbf{N}$ & Mean & $\begin{array}{l}\text { Std. } \\
\text { Deviation }\end{array}$ & $\begin{array}{l}\text { Std. } \\
\text { Error }\end{array}$ & $\begin{array}{l}95 \% \\
\text { Confidence } \\
\text { Interval for } \\
\text { Mean } \\
\text { Lower Bound }\end{array}$ & $\begin{array}{l}\text { Upper } \\
\text { Bound }\end{array}$ & Max & $\begin{array}{l}\mathbf{p} \\
\text { value }\end{array}$ & \\
\hline \multirow{4}{*}{$\begin{array}{l}\text { Left Na } \\
1 \\
\text { Vitreous } \\
\text { humour }\end{array}$} & $0-12$ hrs & 53 & 139.74 & 9.38 & 1.29 & 137.15 & 142.33 & 126.30 & 158.00 & \multirow[t]{4}{*}{$\mathrm{p}<0.001$} \\
\hline & $12-24 \mathrm{hrs}$ & 40 & 145.39 & 7.71 & 1.22 & 142.92 & 147.85 & 124.80 & 159.00 & \\
\hline & Above $24 \mathrm{hrs}$ & 7 & 137.54 & 7.34 & 2.78 & 130.75 & 144.33 & 126.00 & 148.10 & \\
\hline & Total & 100 & 141.85 & 9.04 & .90 & 140.05 & 143.64 & 124.80 & 159.00 & \\
\hline \multirow{4}{*}{$\begin{array}{l}\text { Left K } 1 \\
\text { Vitreous } \\
\text { humour }\end{array}$} & $0-12 \mathrm{hrs}$ & 53 & 6.92 & 2.86 & .39 & 6.14 & 7.71 & 3.10 & 18.70 & \multirow[t]{4}{*}{$\mathrm{p}<0.001$} \\
\hline & $12-24 \mathrm{hrs}$ & 40 & 8.76 & 1.76 & .28 & 8.19 & 9.32 & 6.30 & 14.90 & \\
\hline & Above $24 \mathrm{hrs}$ & 7 & 10.64 & 3.40 & 1.29 & 7.49 & 13.79 & 6.90 & 14.90 & \\
\hline & Total & 100 & 7.92 & 2.75 & .27 & 7.37 & 8.46 & 3.10 & 18.70 & \\
\hline \multirow{4}{*}{$\begin{array}{l}\text { Right } \\
\text { Na1 } \\
\text { Vitreous } \\
\text { humour }\end{array}$} & $0-12 \mathrm{hrs}$ & 53 & 139.66 & 9.51 & 1.31 & 137.04 & 142.28 & 126.50 & 157.90 & \multirow[t]{4}{*}{$\mathrm{p}<0.001$} \\
\hline & $12-24 \mathrm{hrs}$ & 40 & 145.27 & 7.77 & 1.23 & 142.79 & 147.76 & 125.10 & 158.70 & \\
\hline & Above $24 \mathrm{hrs}$ & 7 & 137.36 & 7.32 & 2.77 & 130.58 & 144.13 & 125.70 & 147.90 & \\
\hline & Total & 100 & 141.75 & 9.12 & .91 & 139.93 & 143.56 & 125.10 & 158.70 & \\
\hline \multirow{4}{*}{$\begin{array}{l}\text { Right K } \\
1 \\
\text { Vitreous } \\
\text { humour }\end{array}$} & $0-12 \mathrm{hrs}$ & 53 & 6.94 & 2.86 & .39 & 6.15 & 7.73 & 2.90 & 18.60 & \multirow[t]{4}{*}{$\mathrm{p}<0.001$} \\
\hline & $12-24 \mathrm{hrs}$ & 40 & 8.91 & 1.76 & .28 & 8.34 & 9.47 & 5.80 & 14.60 & \\
\hline & Above $24 \mathrm{hrs}$ & 7 & 11.11 & 3.75 & 1.42 & 7.65 & 14.58 & 7.20 & 16.30 & \\
\hline & Total & 100 & 8.02 & 2.83 & .28 & 7.46 & 8.58 & 2.90 & 18.60 & \\
\hline \multirow{4}{*}{$\begin{array}{l}\text { Left Na } \\
2 \\
\text { Vitreous } \\
\text { humour }\end{array}$} & $0-12 \mathrm{hrs}$ & 31 & 140.8 & 7.3 & 1.3 & 138.1 & 143.4 & 131.0 & 156.4 & \multirow[t]{4}{*}{$\mathrm{p}<0.001$} \\
\hline & $12-24 \mathrm{hrs}$ & 56 & 146.6 & 6.9 & 0.9 & 144.7 & 148.4 & 128.3 & 165.3 & \\
\hline & Above $24 \mathrm{hrs}$ & 12 & 140.2 & 6.8 & 2.0 & 135.9 & 144.6 & 126.3 & 149.2 & \\
\hline & Total & 99 & 144.0 & 7.6 & 0.8 & 142.5 & 145.5 & 126.3 & 165.3 & \\
\hline \multirow{4}{*}{$\begin{array}{l}\text { Left K } \\
2 \\
\text { Vitreous } \\
\text { humour }\end{array}$} & $0-12 \mathrm{hrs}$ & 31 & 7.6 & 1.8 & 0.3 & 7.0 & 8.3 & 5.2 & 13.9 & \multirow[t]{4}{*}{$\mathrm{p}<0.001$} \\
\hline & $12-24 \mathrm{hrs}$ & 57 & 9.8 & 2.1 & 0.3 & 9.2 & 10.4 & 6.8 & 18.9 & \\
\hline & Above $24 \mathrm{hrs}$ & 12 & 12.5 & 3.3 & 1.0 & 10.4 & 14.6 & 7.5 & 17.7 & \\
\hline & Total & 100 & 9.5 & 2.7 & 0.3 & 8.9 & 10.0 & 5.2 & 18.9 & \\
\hline \multirow{4}{*}{$\begin{array}{l}\text { Right } \\
\mathrm{Na} 2 \\
\text { Vitreous } \\
\text { humour }\end{array}$} & $0-12 \mathrm{hrs}$ & 31 & 140.6 & 7.1 & 1.3 & 138.0 & 143.2 & 130.6 & 156.7 & \multirow[t]{4}{*}{$\mathrm{p}<0.001$} \\
\hline & $12-24 \mathrm{hrs}$ & 57 & 146.3 & 7.0 & 0.9 & 144.5 & 148.2 & 128.5 & 165.8 & \\
\hline & Above $24 \mathrm{hrs}$ & 12 & 139.6 & 7.5 & 2.2 & 134.9 & 144.4 & 126.8 & 149.4 & \\
\hline & Total & 100 & 143.8 & 7.7 & 0.8 & 142.2 & 145.3 & 126.8 & 165.8 & \\
\hline \multirow{4}{*}{$\begin{array}{l}\text { Right K } \\
2 \\
\text { Vitreous } \\
\text { humour }\end{array}$} & $0-12 \mathrm{hrs}$ & 31 & 7.7 & 1.9 & 0.3 & 7.0 & 8.4 & 4.9 & 14.7 & \multirow[t]{4}{*}{$\mathrm{p}<0.001$} \\
\hline & $12-24 \mathrm{hrs}$ & 57 & 10.0 & 2.2 & 0.3 & 9.4 & 10.5 & 6.8 & 19.1 & \\
\hline & Above $24 \mathrm{hrs}$ & 12 & 12.9 & 3.5 & 1.0 & 10.7 & 15.1 & 7.9 & 18.1 & \\
\hline & Total & 100 & 9.6 & 2.8 & 0.3 & 9.1 & 10.2 & 4.9 & 19.1 & \\
\hline
\end{tabular}

and time since death were significantly correlated $(\mathrm{r}=0.450)$, $(r=0.421),(r=0.574),(r=0.567)$

Table 4 shows the Pearson coefficient of 0.580 of sample I of right vitreous potassium, 0.536 of sample I of left vitreous potassium, 0.611 of sample II of right vitreous potassium and 0.581 of sample II of left vitreous potassium which has a significant correlation with time since death whereas sodium has negative correlation.

Table 5 shows the comparison of sodium and potassium values of sample 1 and 2 of right and left vitreous. Statistical analysis shows $\mathrm{P}>0.05$ which concludes there is no significant difference between right and left values.

Table 6 shows the $T$ test which compares the values of sodium and potassium of sample 1 and 2 of right and left vitreous with timing 0-12 hours,12-24 hours, and more than 24 hours. Statistical analysis shows $\mathrm{p}>0.05$ for sodium values which is not significant whereas $\mathrm{p}<0.05$ for potassium is significant. which concludes there is no significant difference.

Table 7 shows the one way ANOVA for comparing sodium and potassium values of both sides for three periods in sample I and sample 2 respectively. It shows $\mathrm{p}<0.001$ which is a statistically significant data which concludes that there is a linear correlation of increase in potassium with time since death.

\section{Conflicts of interest}

All contributing authors declare no conflicts of interest. 


\section{Source of Funding}

None.

\section{References}

1. Parikh CK. 2000.

2. Dr, Subrahmanyam's. Law publishers (India) Pvt Ltd. 2001;.

3. Dr KS, Reddy N. CBS Publishers and Distributors.

4. Aggarwal RL, Gupta PC, Nagar CK. Determination of time of death by estimating potassium level in the cadaver vitreous humour. Indian Journal of Ophthalmology. 1983;31(5):528-531.

5. Donaldson AE, Lamont IL. Biochemical changes that occur after death: Potential markers for Determining Post mortem. Interval PloS One. 2013;8(11):82011-82011.

6. Vij K. 2002.

7. Madea B, Musshoff F. Postmortem biochemistry. Elsevier BV; 2007. Available from: https://dx.doi.org/10.1016/j.forsciint.2006.05. 023. do1:10.1016/].forsciint.2006.05.023.

8. Coe JI. Vitreous potassium as a measure of the postmortem interval: An historical review and critical evaluation. Forensic Science International. 1989;42(3):201-213. Available from: https: //dx.doi.org/10.1016/0379-0738(89)90087-x. 1010.106/0379 $0738(89) 90087-x$.

9. Khurana AK

10. Jacobeic A, Fredrick.

11. Saugstad OD, Olaisen B. Post-mortem hypoxanthine levels in the vitreous humour an introductory report. Forensic Science International. 1978;12(1):33-36. Available from: https://dx.doi.org/ 10.1016/0379-0738(78)90031-2. đoi:10.1016/0379-0738(78)90031D.

12. Sturner WQ, Gantner GE. The Postmortem Interval. American Journal of Clinical Pathology. 1964;42(2):137-144. Available from: https://dx.doi.org/10.1093/ajcp/42.2.137. do1:10.1093/ajcp/42.2.137.

13. Singh D, Prashad R, Parkash C, Bansal YS, Sharma SK, Pandey AN. Linearization of the relationship between serum sodium, potassium concentration, their ratio and time since death in Chandigarh zone of north-west India. Elsevier BV; 2002. Available from: https://dx.doi.org/10.1016/s0379-0738(02)00267-0. doi:10.1016/s0379-0738(02)00267-0.

14. Research Fellow - Analysis of sodium and potassium in vitreous humour have been studied to estimate time since death. Double logarithmic, linear relationship between postmortem vitreous Sodium/Potassium electrolytes concentration ratio and time since death in subjects of Chandigarh zone of northwest India. Senior Lecturer, Mr Pandey Avadh Naresh1. 2005;p. 971-0973.

15. Chandrakanth HV, Kanchan T, Balaraj BM, Virupaksha HS, Chandrashekar TN. Postmortem vitreous chemistry - An evaluation of sodium, potassium and chloride levels in estimation of time since death (during the first $36 \mathrm{~h}$ after death). Journal of Forensic and Legal Medicine. 2013;20(4):211-216. Available from: https://dx.doi.org/10. 1016/j.jflm.2012.09.001. doi:10.1016/].jHm.2012.09.001.

16. Balasooriya BAW, Hill CAS, Williams AR. The biochemistry of vitreous humour. A comparative study of the potassium, sodium and urate concentrations in the eyes at identifical time intervals after death. Elsevier BV; 1984. Available from: https://dx.doi.org/10.1016/03790738(84)90064-1. d01:10.1016/0379-0738(84)90064-1.

17. Dr. Vishal Garg -changes in the levels of vitreous potassium with increasing time since death. JIAFM. 2004;26(4).

18. Sturner WQ, E G. Ganter - The postmortem interval; a study of potassium in the vitreous humour. Am J Clin Pathol. 1964;42(2):137144.

19. H WM. Hughes-Levels of potassium in the vitreous humour after death. Med Sci Law. 1965;5:150-156.

20. Hansson L, Uotila U, Lindfors R, K. Laiho Potassium content of the vitreous body as an aid in determining the time of death. J Forensic Sci. 1966;11(3):390-393.
21. J. Lie - Changes of potassium concentration in the vitreous humour after death. Am J Med Sci. 1967;254:136-146.

22. Leahy MS, R E. Farber - Postmortem chemistry of human vitreous humour. J Forensic Sci. 1969;12:214-222.

23. Madea B, Henssge C, Honig W, A. Gerbracht - References for determining the time of death by postmortem vitreous humour. Forensic Sci Int. 1989;40:231-243.

24. Madea B, Kreuser C, S. Banaschak - Postmortem biochemical examination of synovial fluid-a preliminary study. Forensic Sci Int. 2001;118:29-35.

25. J. Coe - Use of chemical determinations on vitreous humour in forensic pathology. J Forensic Sci. 1972;17:541-546.

26. Swift PGF, Worthy E, Emery JL. Biochemical state of the vitreous humour of infants at necropsy. BMJ; 1974. Available from: https: //dx.doi.org/10.1136/adc.49.9.680. 10ل:10. 136/adc.49प.680

27. Komura S, S. Oshtro - Potassium levels in the aqueous and vitreous humor after death. Tohoku J Exp Med. 1977;122:65-68.

28. J. Coe - Postmortem chemistry of blood cerebrospinal fluid and vitreous humor-Tedeschi. vol. II. Company WBS, editor; 1977.

29. Blumenfeld TA, Mantell $\mathrm{CH}$, Catherman RL, A W. Blank Postmortem vitreous humour chemistry in sudden infant death syndrome and in other causes of death in childhood. Am J Clin Pathol. 1979;71:219-223.

30. Choo-Kang E, Mckoy C, C. Escoffery - Vitreous humor analytes in assessing the postmortem interval and the antemortem clinical status. West Indian Med J. 1983;32:23-23.

31. Farmer JG, Benomran, Watson WA. Harland - Magnesium,potassium, sodium and calcium in postmortem vitreous humour from humans. Forensic Sci Int. 1985;27:1-13.

32. Devgun MS, A J. Dunbar - Biochemical investigation of vitreous; application in forensic medicine, especially in relation to alcohol. Forensic Sci Int. 1986;31:27-34.

33. Stephen RJ, G K. Richards- Vitreous humour chemistry. The use of potassium concentration for the prediction of postmortem interval. $J$ Forensic Sci. 1987;32(2):503-509.

34. Sparks DL, Oeltgen PR, Kryscio RJ, L J. Hunsaker -Comparison of chemical methods for determination of postmortem interval. $J$ Forensic Sci. 1989;34(1):197-206.

35. H A. Singh -Potassium concentration analysis in vitreous humour for estimation of time of Death. J Forensic Med Toxicol. 1999;11(3-4):12_ 16.

36. Lange N, Swearer S, Q W. Sturner - Human postmortem interval estimation from vitreous potassium; an analysis of original data from six different studies. Forensic Sci Int. 1994;66:159-174.

37. Knight B. The use of vitreous humour chemistry in timing death, Forensic pathology. Oxford University Press Inc; 1996. p. 91-94.

38. Govekar G, Bishnukumar PC, Dikshit TK. Mishra - Study of potassium in vitreous in relation to death interval and cause of death J Forensic Med Toxicol. 1996;4(1):26-28.

39. James RA, Hoadley BG. Sampson - Determination of postmortem interval by sampling vitreous humour - Am. J Forensic Med Pathol. 1997;18(2):158-162.

40. Pounder DJ, Carson DO, Johnston K, Y. Orihara -Electrolyte concentration differences between left and right vitreous humor samples. J Forensic Sci. 1998;43(3):604-607.

41. Chaudhary BL, Veena M, H B. Tirpude - Potassium concentration in vitreous humour in relation to death interval. J Forensic Med Toxicol. 2007;24(1):26-30.

42. A N. Sheikh - Estimation of potassium interval according to time course of potassium ion activity in cadaveric synovial fluid - Indian. $J$ Forensic Med Toxicol. 2007;1:45-49.

43. Siddhamsetty AK, Verma SK, Kohli A, Verma A, Puri D, Singh A Exploring time of death from potassium, sodium, chloride, glucose \& calcium analysis of postmortem synovial fluid in semi arid climate. Elsevier BV; 2014. Available from: https://dx.doi.org/10.1016/j.jflm. 2014.09.004. do1:10.1016/1.jHm.2014.09.004

44. William MH. 1992. 


\section{Author biography}

S. Angayarkanni, Assistant Professor
Cite this article: Angayarkanni S. Analysis of vitreous humour in determining postmortem interval (time since death) - A prospective study. IP Int J Forensic Med Toxicol Sci 2020;5(4):121-129. 